\title{
Sensorimotor Strategies in Individuals With Poststroke Hemiparesis When Standing Up Without Vision
}

\author{
Yuko Kuramatsu Yuji Yamamoto \\ Tohoku University Nagoya University
}

\author{
Shin-Ichi Izumi \\ Tohoku University
}

\begin{abstract}
This study investigated the sensorimotor strategies for dynamic balance control in individuals with stroke by restricting sensory input that might influence task accomplishment. Sit-to-stand movements were performed with restricted vision by participants with hemiparesis and healthy controls. The authors evaluated the variability in the position of participants' center of mass and velocity, and the center-of-pressure position, in each orthogonal direction at the lift-off point. When vision was restricted, the variability in the mediolateral center-of-pressure position decreased significantly in individuals with hemiparesis, but not in healthy controls. Participants with hemiparesis adopted strategies that explicitly differed from those used by healthy individuals. Variability may be decreased in the direction that most requires accuracy. Individuals with hemiparesis have been reported to have asymmetrical balance deficits, and that meant they had to prioritize mediolateral motion control to prevent falling. This study suggests that individuals with hemiparesis adopt strategies appropriate to their characteristics.
\end{abstract}

Keywords: center of pressure, dynamic balance control, falling, motor learning, whole-body movement

The sensorimotor system makes continual adjustments to allow humans to move easily despite an unstable body structure (Todorov, 2004). It functions not only in healthy people but also in patients with hemiparesis who have to cope with additional challenges (Reisman, Wityk, Silver, \& Bastian, 2007). Strokes can result in sensory impairment, muscle weakness, and loss of dexterity on one side of the body (Adams, Gandevia, \& Skuse, 1990; Canning, Ada, \& O’Dwyer, 2000).

Kuramatsu and Izumi are with the Department of Physical Medicine and Rehabilitation, Graduate School of Medicine, Tohoku University, Sendai, Japan. Izumi is also with the Graduate School of Biomedical Engineering, Tohoku University, Sendai, Japan. Yamamoto is with the Research Center of Health, Physical Fitness and Sports, Nagoya University, Nagoya, Japan. Kuramatsu (kuramatsuy@med. tohoku.ac.jp) is corresponding author. 
Humans, being bipeds, are inherently unstable because a large body mass is held erect with the center of mass (CoM) located high above a relatively small support base (Latash, 2002). Therefore, the asymmetric sensorimotor difficulties associated with poststroke hemiparesis, which can result in weak motor function, disordered coordination (Tseng \& Morton, 2010), and problems with balance (Genthon et al., 2008), may dramatically affect daily activities. Under these circumstances, patients with hemiparesis must develop dynamic balance control so that the timing and spatial configuration of their movements are effective (Carr \& Shephard, 2003). However, it is unclear whether a strategy for solving this problem exists in individuals with hemiparesis.

A study on the dynamic balance control of whole-body movements in healthy individuals conducted by Bauby and Kuo (2000) found that lateral variations were larger than fore-aft variations in gait foot placement, suggesting that stability was influenced by direction. Wurdeman, Huben, and Stergiou (2012) reported that stability during motion, which was measured by variability in step length or width, depends on the general direction of progression. As a result, it is the anteroposterior direction that is primarily controlled during locomotion. Kuramatsu, Muraki, Oouchida, Sekiguchi, and Izumi (2012) analyzed the whole-body movements in the sit-to-stand (STS) transfer when visual and somatosensory inputs were restricted. They also demonstrated that variations in CoM velocity significantly decreased in the major directions of motion (i.e., the anteroposterior and vertical directions) during a critical control period of the STS movement (the liftoff period). Additionally, Reisman, Scholz, and Schoner (2002) suggested that anteroposterior control was more important than vertical control for successful STS performance. They derived a mathematical model that relates body segment angles and their angular velocities to CoM angular and linear momentum, using the uncontrolled manifold hypothesis in Bernstein's degrees of freedom problem (Bernstein, 1967; Scholz \& Schoner, 1999; Scholz, Reisman, Schoner, 2001). They found that when standing up under more challenging conditions, variability increased most strongly for those angles and angular velocity combinations that left CoM momentum invariant, whereas smaller increases were registered for combinations that affected CoM momentum. Additionally, this finding was much more pronounced in the anteroposterior direction than in the vertical direction. According to the uncontrolled manifold hypothesis, the successful accomplishment of a motor task depends on stabilizing a time series of variables (e.g., CoM momentum) that is important for the successful performance of the task. In the above-mentioned study, the configurations of the motor elements that led to a change in the value of a task variable (e.g., CoM momentum) were controlled (i.e., variability must be restricted or stabilized), whereas the configurations of the elements that were consistent with the desired values of the task variable were free from control. Therefore, the anteroposterior direction (i.e., the advancing direction of whole-body movements that include walking and standing up) should be more strongly controlled than the mediolateral direction.

However, individuals with hemiparesis have impairments that affect one side of the body. They may be unable to balance their body weight equally on each foot, and this causes a greater fall risk on the paretic side during whole-body movements (Kajrolkar \& Bhatt, 2016). Therefore, prioritizing mediolateral dynamic balance control might have different implications for individuals with hemiparesis 
compared with healthy individuals. Previous studies on dynamic balance control demonstrated that the paretic side had a weaker response to perturbations (de Kam, Kamphuis, Weerdesteyn, \& Geurts, 2017), and that mediolateral gait instability was greater (Kao, Dingwell, Higginson, \& Binder-Macleod, 2014) in poststroke people compared with healthy individuals. Other studies have investigated correlations between clinical scores and laboratory-based measures, including marginof-stability (Vistamehr, Kautz, Bowden, \& Neptune, 2016) and whole-body angular momentum (Nott, Neptune, \& Kautz, 2014), and examined the effect of visual feedback on gait in individuals with chronic stroke (Walker, Hyngstrom, $\&$ Schmit, 2016). However, it is unclear how individuals with hemiparesis adapt to accommodate both the major direction of motion (anteroposterior or vertical) and the mediolateral direction that is inherently unstable for them.

We investigated dynamic balance control in poststroke individuals with hemiparesis executing whole-body movements to try to understand the sensorimotor adaptations that may have developed. STS is a mechanically demanding, daily, whole-body activity (Carr \& Shephard, 2003). Controlling both the CoM and the center of pressure (CoP) is important during STS, particularly at the lift-off point when the body mass is raised from a stable support to a position of lower mechanical stability (Schenkman, Berger, Riley, Mann, \& Hodge, 1990). In contrast to healthy individuals, individuals with hemiparesis may need to control mediolateral instability at the lift-off point because they tend to bear weight on the unaffected leg with minimal support from the affected leg. Strategies for controlling instability have been investigated by imposing perturbations or restricting sensory inputs that might influence task accomplishment (Reisman et al., 2002). However, multiple inputs that include visual, somatosensory, and vestibular systems have redundant/complementary roles in perceiving postural orientation (Horak \& Macpherson, 1996) and can transform the unstable human body into a controllable system (Bernstein, 1996). In particular, individuals with hemiparesis are reportedly heavily reliant on visual inputs (Marigold \& Eng, 2006). A whole-body movement task can be constrained by restricting the vision of poststroke individuals with hemiparesis, who then have to adjust their movements to complete the task. Therefore, participants were asked to perform the STS with and without the aid of vision. Variations in the CoM and CoP were analyzed to evaluate how well these were regulated when vision was restricted. In this study, the concept of stability, from the perspective of control theory as suggested by Schoner (1995), was applied to investigate the strategies employed by individuals with hemiparesis. Stability was defined as the persistence of a particular state in the face of (phasic) perturbations. Based on this concept, stability can be assessed experimentally using the reproducibility of the variable from trial to trial, such as kinematics at matching points in time during movement (Schoner, 1990), or using the variability of the corresponding variable in time, such as the fluctuation of a fixed point for postural states (Scholz \& Kelso, 1989; Scholz, Kelso, \& Schoner, 1987). These are assumed to assess nervous system control. The less the variation of the measured variable (i.e., more stable), the more the central nervous system controls that variable (Schoner, 1995). We hypothesized that variations in the position and velocity of the CoM and in the position of the $\mathrm{CoP}$ in the mediolateral direction would decrease when vision was restricted at the lift-off point in individuals with hemiparesis, but not in controls. 
Also, we hypothesized that individuals with hemiparesis would be more affected than controls when vision was constrained.

\section{Methods}

\section{Participants}

Eight individuals with hemiparesis (two women and six men; $M \pm S D$, age $58.0 \pm$ 6.1 years; $>6$ months poststroke) and eight healthy individuals (four women and four men; age $=57.8 \pm 5.6$ years) participated in this study. The average weights and heights of the hemiparetic and control groups were $62.4 \pm 9.0 \mathrm{~kg}$ and $1.65 \pm$ $0.08 \mathrm{~m}$, and $60.4 \pm 12.4 \mathrm{~kg}$ and $1.62 \pm 0.10 \mathrm{~m}$, respectively. Participants with stroke exhibited clinical symptoms consistent with lower-extremity hemiparesis caused by a unilateral hemorrhagic brain lesion. They were able to rise from a chair with confidence without using their arms and were also able to walk $4 \mathrm{~m}$ without a prosthesis. Exclusion criteria were a significant cognitive deficit (Folstein MiniMental Examination score <26/30; Folstein, Folstein, \& McHugh, 1975), evidence of nonstroke-related disabilities, and unilateral spatial neglect evaluated using the line bisection task (Liepman \& Kalmus, 1900). None of the participants with hemiparesis had hemianopia or visual problems of daily activities. Participants with hemiparesis were evaluated during physical examinations according to the following parameters to assess their motor impairment, muscle tone, sensitivity to touch, positional sensitivity, and balance: Brunnstrom stage (Brunnstrom, 1966), stroke impairment assessment set (Chino, Sonoda, Domen, Saitoh, \& Kimura, 1994; Tsuji, Liu, Sonoda, Domen, \& Chino, 2000), and Berg Balance Scale (Berg, Wood-Dauphinee, \& Williams, 1995). The participants had mild to moderate motor impairment for lower-extremity motor functions on the stroke impairment assessment set (hip flexion, knee extension, and foot-pad). Two participants exhibited severe hypesthesia of a tactile and position sensation of the foot, while two others exhibited light hypesthesia of a tactile or position sensation. The remaining participants exhibited good tactile and position sensations, presenting moderate spasticity at the ankle for muscle tone. On the Berg Balance Scale, one participant scored 30 (moderately severe impairment), while all other participants scored between 42 and 56 (two participants were moderately impaired; five participants were lightly impaired) (Table 1). Regarding asymmetrical balance in individuals with hemiparesis, six poststroke participants needed foot or leg braces for daily activities on the affected side and two participants who did not require a gait-aid (i.e., foot or leg brace) walked also with a limp due to reduced weight-bearing ability in the affected leg. None of the healthy controls had a neurological disease or visual problems. All participants provided written consent before participating in these experiments, which were approved by the institutional ethics committee at Tohoku University.

\section{Equipment and Setup}

The MAC 3D System (Motion Analysis Corp., Santa Rosa, CA) motion measurement device and four force plates (Anima Corp., Tokyo, Japan) were used to collect the experimental data. The system was comprised of eight infrared video 


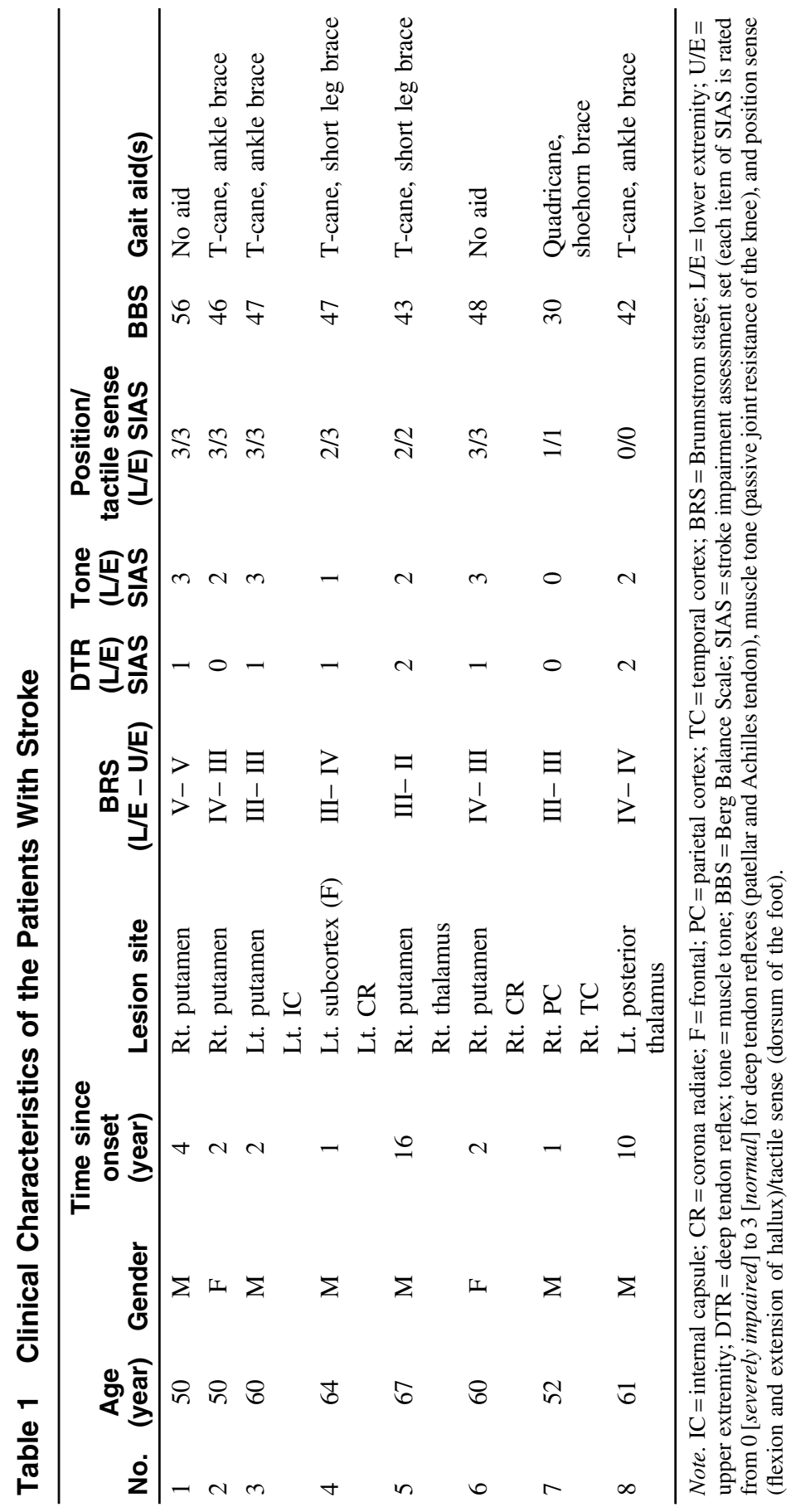


cameras suspended from the ceiling; the error in the measurement volume was $500 \pm 0.9 \mathrm{~mm}$ for all cameras (length $4,000 \mathrm{~mm}$; width $4,000 \mathrm{~mm}$; and height $2,000 \mathrm{~mm}$ ). The $x-, y$-, and $z$-axes were the mediolateral, anteroposterior, and vertical directions, respectively. The positive directions were defined as right, anterior, and upward. Reflective markers were attached to the following anatomical landmarks: the base of the fifth metatarsal in both feet, both lateral malleoli, both lateral femoral condyles, both hip joints (at one-third the distance from the greater trochanter to the anterior superior iliac spine), the anterior superior iliac spine, the posterior superior iliac spine, both acromion processes, the right scapula (a dummy was positioned to distinguish the right from the left side), the spinous process of the second thoracic vertebra, the point anterior to the external auditory meatus on both sides, and the glabella. A stool was placed on both of the rear force plates to aid separate foot placement on the front force plates. The size of the seating surface set horizontally was $400 \mathrm{~mm}$ in length and $500 \mathrm{~mm}$ in width, and its height was adjustable. Data from the video cameras and force plates were sampled at $120 \mathrm{~Hz}$ and synchronized.

\section{Experimental Procedure}

Participants performed the STS movement barefoot 20 times each with their eyes closed or open. The movements were performed in two different orders (either $\mathrm{ABAB}$ or $\mathrm{BABA}$ ) that were assigned equally among the participants to counterbalance fatigue and practice effects: A, STS five times with eyes open; B, STS five times with eyes closed. In other words, four participants with hemiparesis and four controls performed five trials with eyes open at first, then five trials with eyes closed, then five trials with eyes open, and finally, five trials with eyes closed. The other set of participants began with the eye-closed condition. The initial sitting posture was standardized for all repetitions-with the height of the seating surface matched to the fibular head of each participant. The distance between the heels of each participant was maintained at two-thirds of the full length of the participant's foot depending on their own ease with the movement. Approximately half of the thigh was in contact with the stool, and the knees were flexed at approximately $100^{\circ}$. The position of the buttocks on the seat and the position of each foot on the force platform were marked with tape, and prior to each trial, each participant's starting position was adjusted to be in alignment with these marks. Participants were instructed to hang their arms loosely at their sides, and they were not allowed to make contact with the stool during the STS.

Before measurements were made, participants were instructed to move naturally from a sitting to a standing position at their preferred speed. Immediately after the participant had assumed a seated posture and felt vertical, measurements were initiated by sounding a buzzer. After each participant maintained a sitting position for approximately $8 \mathrm{~s}$, a second buzzer sounded as the initiation signal for the movement. The participants were told that this was not a reaction-time task and to stand up in a comfortable and natural manner without hesitation after the buzzer sound. Participants maintained their posture after standing up until a third buzzer sounded $8 \mathrm{~s}$ later. Participants were allowed to take rest between trials, if they felt tired. After all experimental repetitions, participants were asked to describe any differences, difficulties they encountered, or anxiety they experienced during STS 
under the two conditions. Falls were prevented by an experimenter monitoring the movements at the participant's side.

\section{Data Reduction and Analysis}

The coordinates of each marker were passed through a low-pass filter with a cut-off frequency of $6 \mathrm{~Hz}$ and recorded using EVaRT motion capture software (version 5.0.4; Motion Analysis Corp.). The overall CoM locations were calculated based on body segment length (Winter, 1990). The position and velocity of the CoM and the force plate signals were passed through a low-pass filter at $5 \mathrm{~Hz}$. We used KineAnalyzer analytical software (version 3.0.0.606; Kissei Comtec Co., Ltd., Nagano, Japan) for data reduction.

Figure 1 presents a schematic time series of one typical trial showing the STS movement with $\mathrm{CoP}$ and $\mathrm{CoM}$ kinematics. The anteroposterior $(y)$ and mediolateral $(x)$ displacements of the CoP were calculated using the following equations:

$$
\mathrm{CoP} x=[-\mathrm{M} y+(h * \mathrm{~F} x)] / \mathrm{F} z,
$$

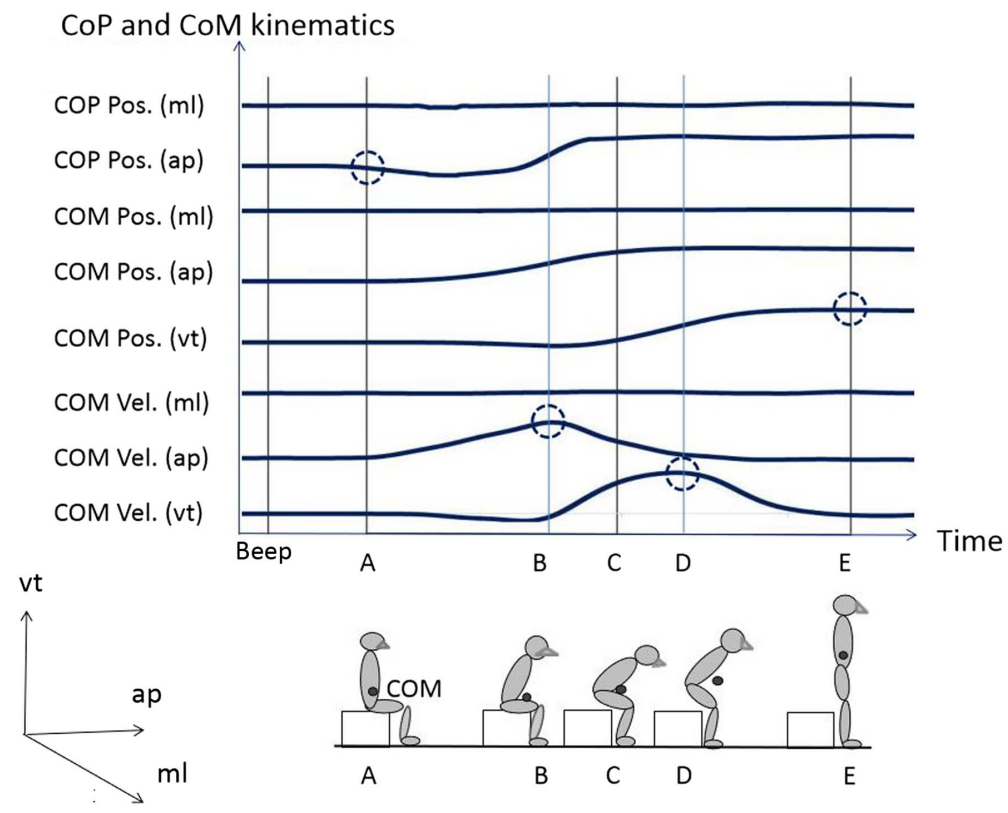

Figure 1 - Schematic time series of center of pressure (CoP) and center of mass (CoM) kinematics. CoP (positions in the mediolateral and anteroposterior directions) and CoM (position and velocity in the mediolateral, anteroposterior, and vertical directions); the $\mathrm{ml}$ axis is in the mediolateral direction; the ap axis is in the anteroposterior direction; and the vt axis is in the vertical direction. Right, anterior, and upward directions are defined as positive directions. The period A-E is defined as the sit-to-stand (STS) movement time: A, STS initiated; B, peak velocity in the ap direction; C, lift-off; D, peak velocity in the vt direction; and E, STS completed. The movement time is normalized to $100 \%$. 


$$
\mathrm{CoP} y=[\mathrm{M} x+(h * \mathrm{~F} y)] / \mathrm{F} z,
$$

where $h$ is the height of the CoM from the surface of the force platform, Mx and $\mathrm{M} y$ are the moments obtained from the $x$ - and $y$-axes of the force plate, and $\mathrm{F} x$, $\mathrm{Fy}$, and $\mathrm{Fz}$ are the mediolateral, anteroposterior, and vertical ground reaction forces, respectively. Movement onset was defined as backward anteroposterior movement of the CoP (i.e., the first backward excursion of the CoP from the average static sitting position minus $2 S D \mathrm{~s}$ ) as determined by the four force plates. The end of the movement was determined as when the vertical CoM first reached the average plateau standing CoM position, minus $2 S D$ s. The time at which the buttocks lifted from the seat was defined as when the reaction force signal from the stool expired. The movement time (MT) was defined as movement onset to end. The time taken for the movement from onset to the end was normalized to $100 \%$. We compared the MT, the peak anteroposterior and vertical velocities, and the timing of events under both visual conditions in both groups.

We calculated the $S D s$ for all kinematic parameters as measures of variability relative to the CoM and CoP at the lift-off point, which is the critical moment of STS. We calculated SDs for the CoM positions and velocities; the mediolateral, anteroposterior, and vertical; and the mediolateral and anteroposterior $\mathrm{CoP}$ positions over 10 repetitions under each condition.

To evaluate the ability to control $\mathrm{CoM}$ and $\mathrm{CoP}$ with and without vision, variability (i.e., $S D$ ) in the positions and velocities of the CoM and CoP for each direction was subjected to a mixed-design analysis of variance using SPSS software (version 20; SPSS Inc., Chicago, IL) to compare the effects of vision (restricted vision and not restricted vision) and group (individuals with hemiparesis and healthy controls) $(p<.05)$. Interaction effects were assessed and corrected for multiple comparisons using Bonferroni's adjustment. Partial eta squared $\left(\eta_{\mathrm{p}}^{2}\right)$ was used for the evaluation of the effect size.

To illustrate variability in the CoP from movement initiation to the critical control point (lift-off), these measurements time series were shown for each group with two visual conditions. The variability in the CoP position was calculated as the average $S D$ in the CoP positions of the mediolateral and anteroposterior directions of each group.

\section{Results}

\section{Experimental Task Achievement}

No participants appealed for fatigue. Four repetitions were unsuccessful because of two unclear ends of movement (one was a control participant) and failures to complete the eyes-closed movement (light touch to the back and opening of the eyes; participants with hemiparesis nos. 7 and 8). Therefore, data were analyzed from 316/320 repetitions performed by 16 participants.

In terms of MT and peak velocity in the anteroposterior and vertical directions, two-way analysis of variance revealed significant effects of both visual condition and group (effect size was represented by $\eta_{\mathrm{p}}^{2}$ ), such that the MT was longer in the hemiparetic group $\left(F_{1,14}=9.60, p<.01, \eta_{\mathrm{p}}^{2}=.41\right)$ and in the vision-restricted condition $\left(F_{1,14}=7.01, p<.05, \eta_{\mathrm{p}}^{2}=.33\right)$. The movement velocity was lower in 
the hemiparetic group (anteroposterior: $F_{1,14}=7.16, p<.05, \eta_{p}^{2}=.43$; vertical: $F_{1,14}=10.11, p<.01, \eta_{\mathrm{p}}^{2}=.34$ ) and in the vision-restricted condition (anteroposterior: $F_{1,14}=10.39, p<.001, \eta_{\mathrm{p}}^{2}=.43$; vertical: $F_{1,14}=15.51, p<.001, \eta_{\mathrm{p}}^{2}=.53$ ). The interaction effect was significant between visual condition and group in terms of MT $\left(F_{1,14}=5.20, p<.05, \eta_{\mathrm{p}}^{2}=.27\right)$, but not significant for the peak velocities. Post hoc tests revealed a significant difference between the two visual conditions in the hemiparetic group $\left(F_{1,14}=12.14, p<.01, \eta_{\mathrm{p}}^{2}=.46\right)$, but not the control group $\left(F_{1,14}=0.07, p=.79, \eta_{\mathrm{p}}^{2}=.01\right)$ and with regard to MT (Table 2). The effect of constrained vision was apparently greater in participants with hemiparesis than in controls, based on the statistical results for MT and the difference in velocities between the two conditions in each group (Table 2). Table 2 lists the time points of the main STS events: peak velocity of the anteroposterior direction, lift-off, and peak velocity of the vertical direction. MT was normalized to $100 \%$. The event time was variable among participants with hemiparesis, especially in the vision-restricted condition. In this regard, the effects of constrained vision also appeared to be greater in participants with hemiparesis than in controls.

\section{Variability in CoM and CoP}

Figure 2 shows the CoP positions (mediolateral and anteroposterior) and CoM velocities (anteroposterior and vertical) in one control and one participant with hemiparesis (Patient No. 5). The lift-off moment occurred at time zero.

Two-way analysis of variance demonstrated that vision had a significant effect on mediolateral CoP variability at the lift-off moment $\left(F_{1,14}=12.12, p<.01\right.$, $\left.\eta_{\mathrm{p}}^{2}=.46\right)$. The interaction effect between vision/no-vision and group was significant $\left(F_{1,14}=18.20, p<.001, \eta_{\mathrm{p}}^{2}=.57\right)$. Post hoc tests revealed that vision made a significant difference in the hemiparetic group $\left(F_{1,14}=30.01, p<.001, \eta_{\mathrm{p}}^{2}=.68\right)$, but not in the control group $\left(F_{1,14}=0.31, p=.59\right)$. The tests revealed a significant difference between the two groups when vision was not restricted $\left(F_{1,14}=16.78\right.$, $\left.p<.001, \eta_{\mathrm{p}}^{2}=.55\right)$, but not when vision was restricted $\left(F_{1,14}=0.30, p=.59\right)$. Vision $\left(F_{1,14}=5.89, p<.05, \eta_{\mathrm{p}}^{2}=.30\right)$ and group $\left(F_{1,14}=4.61, p<.05, \eta_{\mathrm{p}}^{2}=.25\right)$ both had a significant effect on anteroposterior CoP variability. Figure 3 shows variations in $\mathrm{CoP}$ in the two directions, demonstrating the significant effect of vision (asterisk indicates the interaction effect). In the anteroposterior direction, $\mathrm{CoP}$ variation increased under the vision-restricted condition in both groups, with no interaction, but interaction effects were observed on both visual condition and group in the mediolateral direction. No significant differences were found in the variability of CoM positions or velocities among the three directions in either group, irrespective of whether vision was restricted or not.

Differences in CoP variability between the hemiparetic and control group were observed at the lift-off point depending on whether participant's vision was restricted or not. Figure $4 \mathrm{~A}$ and $4 \mathrm{~B}$ shows the variability time series from movement initiation to lift-off (the horizontal axis shows movement initiation at $0 \%$ and lift-off at $100 \%$ ); these are dynamic processes preceding the control point (lift-off). These figures show that variability in the mediolateral CoP position was not decreased at lift-off in the control group, irrespective of whether vision was restricted or not restricted, and in the hemiparetic group when vision was not 


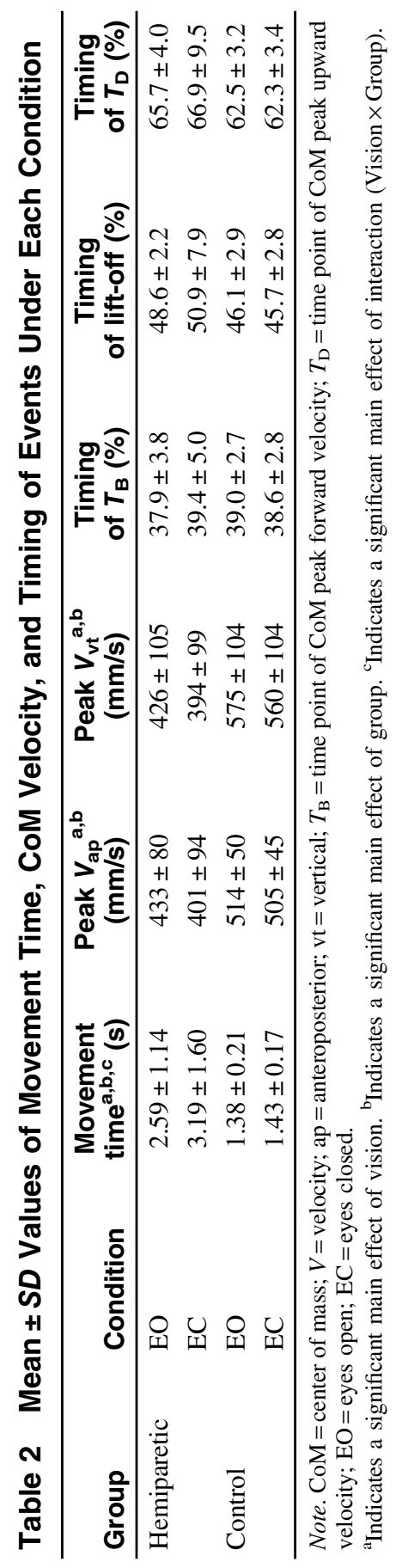




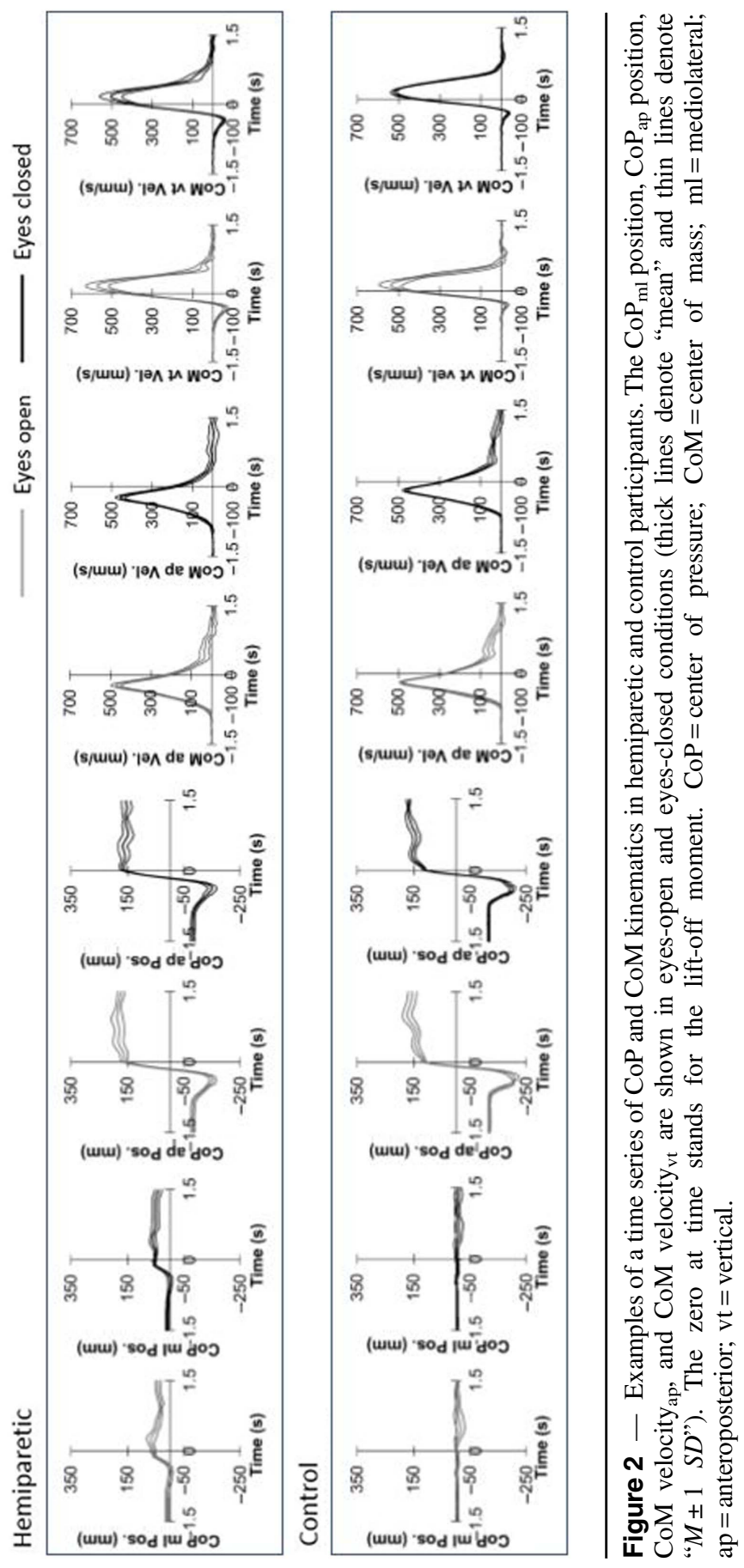



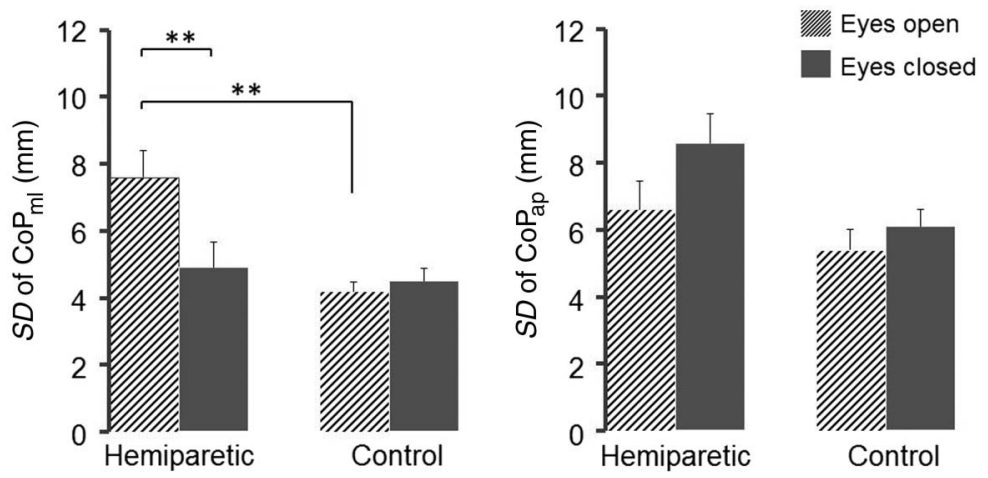

Eyes closed

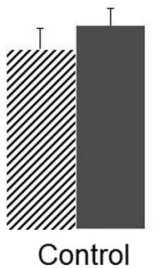

Figure 3 - Variations in CoP at the lift-off point in the two directions, demonstrating the significant effect of vision. The left side shows the mediolateral direction and the right side shows the anteroposterior direction. Error bars represent the standard error. Interaction effects are indicated by asterisks. $\mathrm{CoP}=$ center of pressure; ap $=$ anteroposterior; $\mathrm{ml}=$ mediolateral. $* * p<.001$.
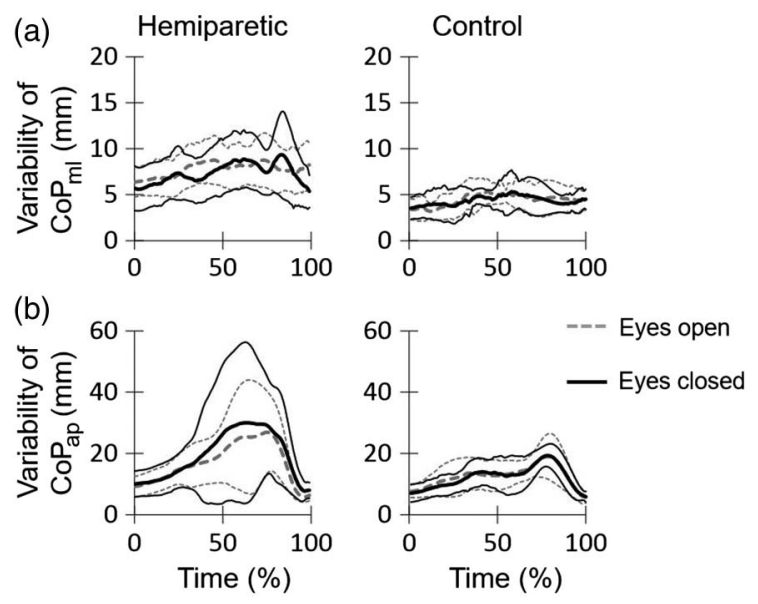

Figure 4 - (a) Variability of the $\mathrm{CoP}$ position in the mediolateral direction from movement initiation toward lift-off. (b) Variability of the CoP position in the anteroposterior direction from movement initiation toward lift-off. The left side shows the hemiparetic group. Thick lines denote "mean," and thin lines denote " $M \pm 1 S D$." The right side shows the control group. $0 \%$ of time represents a movement initiation, and $100 \%$ of time represents a lift-off. $\mathrm{CoP}=$ center of pressure; $\mathrm{ap}=$ anteroposterior; $\mathrm{ml}=$ mediolateral .

restricted. In contrast, variability in the anteroposterior CoP position was decreased at the lift-off point in both groups, irrespective of whether vision was restricted or not restricted, and in the mediolateral CoP position in the hemiparetic group, when vision was restricted. 


\section{Discussion}

This study demonstrated that variability in the mediolateral CoP position decreased significantly when vision was restricted in individuals with hemiparesis, but not in healthy controls. This decreased variability in the CoP when vision was restricted is an important result. Typically, individuals with impairments rely more on vision, so we might expect that removing vision would lead to a disturbance in performance. Mediolateral motion is extremely important for posture control in individuals with hemiparesis. An upright stance and STS transfer in individuals with stroke are reported to be characterized by weight-bearing asymmetry, with more weight being placed on the nonparetic limb (Genthon et al., 2008; Roy et al., 2006). de Kam et al. (2017) reported that postural control was impaired in patients with hemiparesis and suggested an increased risk of falling toward the paretic side. Weight-bearing is typically transferred to the unaffected side in participants with hemiparesis when the body mass is raised from sitting to the less stable standing position during STS movement. Supporting the body weight adequately on both limbs is crucial for maintaining balance when standing (Mansfield, Mochizuki, Inness, \& Mcllroy, 2012). Therefore, enhancing mediolateral balance control may be an effective strategy for individuals with hemiparesis. The present findings suggest that CoP position in the anteroposterior direction was controlled at lift-off in both groups, irrespective of whether vision was restricted or not. In addition, all participants with hemiparesis exhibited a decrease in mediolateral $\mathrm{CoP}$ variability, although different structures had been affected by their strokes, resulting in various sensory and motor impairments. Nevertheless, any individual with hemiparesis exhibits weakness on one side of the body and is unstable in the lateral direction. Consequently, decreasing variability in mediolateral $\mathrm{CoP}$ at the critical control point (i.e., take-off) under constrained conditions might indicate an increase in whole-body movement skill among individuals with hemiparesis. These results appear to be consistent with the optimal feedback control hypothesis (Todorov, 2004; Todorov \& Jordan, 2002). This hypothesis is related to skilled performance; it postulates that under constrained conditions (by enhancing control), variability decreases in the direction that most requires accuracy and that variability is allowed to increase in other directions. The present results demonstrate that variability in the mediolateral CoP position decreases while variability in the anteroposterior CoP position increases in individuals with hemiparesis accompanied by restricted vision. To accomplish the task reliably, those with hemiparesis had to suppress variability in movement in the mediolateral direction, which placed them at higher risk of falls. Being more unstable, they sought to stabilize themselves. Simultaneously, they slowed their motion and tolerated variability in the anteroposterior direction more than controls did. This may not only reflect adaptation but also illustrate the difficulties experienced by individuals with stroke when seeking to prioritize mediolateral control. These individuals were forced to perform the task more cautiously to achieve additional control and took much longer to complete the movement compared with the healthy controls.

No significant decrease in kinematic or kinetic parameter variability was observed in healthy controls when vision was restricted because perception systems can act redundantly, and there is a complementary relationship between the visual and somatosensory systems (Shumway-Cook \& Woollacott, 2007). 
It might be difficult to detect enhanced control in response to perceptual constraints in healthy individuals if only one modality was altered. However, individuals with hemiparesis did show enhanced mediolateral control when only their vision was restricted. Therefore, restricting vision had a more profound effect on individuals with hemiparesis than on healthy controls. It is possible that participants with hemiparesis were unable to use perceptual redundancy and complementarity as effectively as healthy participants. The sensory tests revealed that some participants with hemiparesis had mild to severe somatosensory defects. Others, who showed no somatosensory defects during the test, displayed an exaggerated stretch reflex at the knees and ankles when in the sitting position. Ghosh and Haggard (2014) reported that sensory perception inputs generated as a result of voluntary movements were indistinguishable from those leading to spinal reflexes. Therefore, individuals with hemiparesis might be unable to utilize appropriate somatosensory information during STS movements due to adjustments made by the exaggerated stretch reflex. Chu, Hornby, and Schmit (2015) described a lower response accuracy and higher error rate in dynamic load perception in the lower extremities of individuals with stroke during gait. Another study reported that individuals with hemiparesis did not use proprioceptive information to control balance during gait (Mullie \& Duclos, 2014). In contrast to healthy participants, individuals with hemiparesis might need to enhance balance control when their vision is restricted.

This study did not support the hypothesis that mediolateral variability in the position and velocity of CoM decreased when vision was restricted at lift-off. Only variability in the CoP position decreased at the lift-off point in individuals with hemiparesis. In contrast, our previous study in healthy individuals revealed that variability in CoM velocity did decrease during a given critical period (Kuramatsu et al., 2012). In healthy individuals, CoM velocity may provide better information than CoM position for controlling posture (Jeka, Kiemal, Creath, Horak, \& Peterka, 2004; Masani, Popovic, Nakazawa, Kouzaki, \& Nozaki, 2003; Westlake, Wu, \& Culham, 2007). However, participants with hemiparesis were unable to use the CoM velocity information effectively because of problems that included spasticity, diminished muscular activity, and disruption of the agonist-antagonist sequence associated with volitional motor commands (Gracies, 2005). These symptoms might interfere with motor performance and feedback related to ongoing activities. Therefore, individuals with hemiparesis might be unable to make appropriate adjustments to movement velocity when vision is restricted. When muscular velocity information is unreliable, other somatosensory and vestibular information relating to force, acceleration, and position might compensate and allow a task to be accomplished (Pinsault \& Vuillerme, 2008; Thedon, Mandrick, Foissac, Mottet, \& Perrey, 2011). Instead of continuous spatial control of CoM velocity, individuals with hemiparesis might be able to adjust the CoP position momentarily at the lift-off point and execute motor tasks using positional information and force. In other words, individuals with hemiparesis might use appropriate methods of generating feedback to control STS movements effectively in their own way (Liu \& Todorov, 2007).

This study had a few limitations. First, only STS movements were investigated to clarify strategies for dynamic balance control. Second, a small sample size of eight participants with hemiparesis was used. Despite this small sample size, the 
results revealed that variability in the mediolateral dynamics decreased significantly when vision was restricted (i.e., all individuals with hemiparesis exhibited reduced variability). This parameter had the largest effect size observed in the present study. Future research should include more participants and different measures to confirm the applicability of these findings. For example, a large, heterogeneous group of subjects would have permitted primary analyses on the overall group and secondary analyses on smaller subgroups of subjects homogeneous for certain conditions, allowing for meaningful conclusions to be drawn.

\section{Conclusions}

Participants with hemiparesis with sensorimotor dysfunction on one side of the body adopted movement strategies that differed from those of healthy controls. Under task-constrained conditions, individuals with hemiparesis showed enhanced mediolateral control of the CoP. The characteristics they have in common suggested that they decreased variability in the direction that most requires accuracy and used their own methods of collecting feedback information to control their movements. Individuals with hemiparesis generate strategies for developing their sensorimotor skills to execute tasks as successfully as possible. Therefore, it is inappropriate for patients with hemiparesis to simply imitate the movement patterns of healthy individuals without considering their sensorimotor adaptations. These results demonstrate that a better understanding of patients' sensorimotor strategies is required.

\section{Acknowledgments}

We would like to thank all participating patients; Dr. Shuhei Morise, Dr. Eizaburo Suzuki, Dr. Tomomi Ueda, and Dr. Sumie Uchino for their assistance with data collection; and Dr. Keisetsu Shima, Dr. Haruhiko Satoh, and Dr. Yoshimi Suzukamo for their helpful advice. This study was supported by a Grants-in-Aid for Scientific Research (No. 26350603) (to Y. Kuramatsu) from the Japan Society for the Promotion of Science.

\section{References}

Adams, R.W., Gandevia, S.C., \& Skuse, N.S. (1990). The distribution of muscle weakness in upper motoneuron lesions affecting the lower limb. Brain, 113, 1459-1476. doi:10. 1093/brain/113.5.1459

Bauby, C.D., \& Kuo, A.D. (2000). Active control of lateral balance in human walking. Journal of Biomechanics, 33, 1433-1440. PubMed ID: 10940402 doi:10.1016/S00219290(00)00101-9

Berg, K., Wood-Dauphinee, S., \& Williams, J.I. (1995). The balance scale: Reliability assessment with elderly residents and patients with an acute stroke. Scandinavian Journal of Rehabilitation Medicine, 27, 27-36. PubMed ID: 7792547

Bernstein, N.A. (1967). The coordination and regulation of movements. Oxford, UK: Pergamon.

Bernstein, N.A. (1996). On dexterity and its developments. In M.L. Latash \& M.T. Turvey (Eds.), Dexterity and its development (pp. 3-244). Mahwah, NJ: Lawrence Erlbaum Associates. 
Brunnstrom, S. (1966). Motor testing procedures in hemiplegia: Based on sequential recovery stages. Physical Therapy, 46, 357-375. PubMed ID: 5907254 doi:10. 1093/ptj/46.4.357

Canning, C.G., Ada, L., \& O'Dwyer, N.J. (2000). Abnormal muscle activation characteristics associated with loss of dexterity after stroke. Journal of the Neurological Science, 176, 45-56. doi:10.1016/S0022-510X(00)00305-1

Carr, J.H., \& Shephard, R.B. (2003). Stroke rehabilitation: Guidelines for exercise and training to optimize motor skill. Edinburgh, UK: Butterworth Heinemann.

Chino, N., Sonoda, S., Domen, K., Saitoh, E., \& Kimura, A. (1994). Stroke impairment assessment set (SIAS): A new evaluation instrument for stroke patients. The Japanese Journal of Rehabilitation Medicine, 31, 119-125. doi:10.2490/jjrm1963.31.119

Chu, V.W., Hornby, T.G., \& Schmit, B.D. (2015). Perception of lower extremity loads in stroke survivors. Clinical Neurophysiology, 126, 372-381. PubMed ID: 25097091 doi:10.1016/j.clinph.2014.06.047

de Kam, D., Kamphuis, J.F., Weerdesteyn, V., \& Geurts, A.C.H. (2017). The effect of weight-bearing asymmetry on dynamic postural stability in people with chronic stroke. Gait \& Posture, 53, 5-10. PubMed ID: 28061401 doi:10.1016/j.gaitpost.2016.12.019

Folstein, M.F., Folstein, S.E., \& McHugh, P.R. (1975). "Mini-mental state." A practical method for grading the cognitive state of patients for the clinician. Journal of Psychiatric Research, 12, 189-198. PubMed ID: 1202204 doi:10.1016/0022-3956 (75)90026-6

Genthon, N., Rougier, P., Gissot, A.S., Froger, J., Pelissier, J., \& Perennou, D. (2008). Contribution of each lower limb to upright standing in stroke patients. Stroke, 39, 1793-1799. PubMed ID: 18369174 doi:10.1161/STROKEAHA.107.497701

Ghosh, A., \& Haggard, P. (2014). The spinal reflex cannot be perceptually separated from voluntary movements. The Journal of Physiology, 592, 141-152. doi:10.1113/ jphysiol.2013.260588

Gracies, J.M. (2005). Pathophysiology of spastic paresis. II: Emergence of muscle overactivity. Muscle \& Nerve, 31, 552-571. PubMed ID: 15714511 doi:10.1002/mus.20285

Horak, F.B., \& Macpherson, J.M. (1996). Postural orientation and equilibrium. In J. Shepherd \& L. Rowell (Eds.), Exercise: Regulation and integration of multiple systems, Handbook of physiology, section 12 (pp. 255-292). New York, NY: Oxford University Press.

Jeka, J., Kiemal, T., Creath, R., Horak, F., \& Peterka, R. (2004). Controlling human upright posture: Velocity information is more accurate than position or acceleration. Journal of Neurophysiology, 92, 2368-2379. PubMed ID: 15140910 doi:10.1152/jn.00983.2003

Kajrolkar, T., \& Bhatt, T. (2016). Falls-risk post-stroke: Examining contributions from paretic versus non paretic limbs to unexpected forward gait slips. Journal of Biomechanics, 49, 2702-2708. PubMed ID: 27416778 doi:10.1016/j.jbiomech.2016.06.005

Kao, P., Dingwell, J.B., Higginson, J.S., \& Binder-Macleod, S. (2014). Dynamic instability during post-stroke hemiparetic walking. Gait \& Posture, 40, 457-463. PubMed ID: 24931112 doi:10.1016/j.gaitpost.2014.05.014

Kuramatsu, Y., Muraki, T., Oouchida, Y., Sekiguchi, Y., \& Izumi, S. (2012). Influence of constrained visual and somatic senses on controlling centre of mass during sit-to-stand. Gait \& Posture, 36, 90-94. PubMed ID: 22464270 doi:10.1016/j.gaitpost.2012.01.011

Latash, M.L. (2002). Neurophysiological basis of movement. Champaign, IL: Human Kinetics.

Liepman, H.,\& Kalmus, E. (1900). Über eine Augenmaassstörung bei Hemianopikern. Berliner Klinische Wochenschrift, 38, 838-842.

Liu, D., \& Todorov, E. (2007). Evidence for the flexible sensorimotor strategies predicted by optimal feedback control. Journal of Neuroscience, 27, 9354-9368. doi:10.1523/ JNEUROSCI.1110-06.2007 
Mansfield, A., Mochizuki, G., Inness, E.L., \& Mcllroy, W.E. (2012). Clinical correlates of between-limb synchronization of standing balance control and falls during inpatient stroke rehabilitation. Neurorehabilitation and Neural Repair, 26, 627-635. PubMed ID: 22275158 doi:10.1177/1545968311429688

Marigold, D.S., \& Eng, J.J. (2006). The relationship of asymmetric weight-bearing with postural sway and visual reliance in stroke. Gait \& Posture, 23, 249-255. PubMed ID: 16399522 doi:10.1016/j.gaitpost.2005.03.001

Masani, K., Popovic, M.R., Nakazawa, K., Kouzaki, M., \& Nozaki, D. (2003). Importance of body sway velocity information in controlling ankle extensor activities during quiet stance. Journal of Neurophysiology, 90, 3774-3782. PubMed ID: 12944529 doi:10. $1152 /$ jn.00730.2002

Mullie, Y.,\& Duclos, C. (2014). Role of proprioceptive information to control balance during gait in healthy and hemiparetic individuals. Gait \& Posture, 40, 610-615. PubMed ID: 25127297 doi:10.1016/j.gaitpost.2014.07.008

Nott, C.R., Neptune, R.R., \& Kautz, S.A. (2014). Relationships between frontal-plane angular momentum and clinical balance measures during post-stroke hemiparetic walking. Gait \& Posture, 39, 129-134. PubMed ID: 23820449 doi:10.1016/j. gaitpost.2013.06.008

Pinsault, N., \& Vuillerme, N. (2008). Differential postural effects of plantar-flexor muscle fatigue under normal, altered and improved vestibular and neck somatosensory conditions. Experimental Brain Research, 191, 99-107. PubMed ID: 18663436 doi:10.1007/ s00221-008-1500-z

Reisman, D.S., Scholz, J.P., \& Schoner, G. (2002). Coordination underlying the control of whole body momentum during sit-to-stand. Gait \& Posture, 15, 45-55. PubMed ID: 11809580 doi:10.1016/S0966-6362(01)00158-8

Reisman, D.S., Wityk, R., Silver, K., \& Bastian, A.J. (2007). Locomotor adaptation on a split-belt treadmill can improve walking symmetry post-stroke. Brain, 130, 1861-1872. PubMed ID: 17405765 doi:10.1093/brain/awm035

Roy, G., Nadeau, S., Gravel, D., Malouin, F., McFadyen, B.J., \& Piotte, F. (2006). The effect of foot position and chair height on the asymmetry of vertical forces during sit-tostand and stand-to-sit tasks in individuals with hemiparesis. Clinical Biomechanics, 21, 585-593. doi:10.1016/j.clinbiomech.2006.01.007

Schenkman, M., Berger, R.A., Riley, P.O., Mann, R.W., \& Hodge, W.A. (1990). Wholebody movements during rising to standing from sitting. Physical Therapy, 70, 638-648. doi:10.1093/ptj/70.10.638

Scholz, J.P., \& Kelso, J.A.S. (1989). A quantitative approach to understanding the formation and change of coordinated movement patterns. Journal of Motor Behavior, 21, 122-144. PubMed ID: 15132941 doi:10.1080/00222895.1989.10735470

Scholz, J.P., Kelso, J.A.S., \& Schoner, G. (1987) Nonequilibrium phase transition in coordinated biological motion: Critical slowing down and switching time. Physics Letter A, 123, 390-394. doi:10.1016/0375-9601(87)90038-7

Scholz, J.P., \& Schoner, G. (1999). The uncontrolled manifold concept: Identifying control variables for a functional task. Experimental Brain Research, 126, 289-306. PubMed ID: 10382616 doi:10.1007/s002210050738

Scholz, J.P., Reisman, D.S., \& Schöner, G. (2001) Effects of varying task constraints on solutions to joint coordinationin a sit-to-stand task. Experimental Brain Research, 141, 485-500. PubMed ID: 11810142. doi:10.1007/s002210100878

Schoner, G. (1990). A dynamic theory of coordination of discrete movement. Biological Cybernetics, 63, 257-270. PubMed ID: 2207200 doi:10.1007/BF00203449

Schoner, G. (1995). Recent development and problems in human movement science and their conceptual implications. Ecological Psychology, 7, 291-314. http://e.guigon.free. fr/rsc/article/Schoner95.pdf. doi:10.1207/s15326969eco0704_5 
Shumway-Cook, A., \& Woollacott, M.H. (2007). Motor control: Translating research into clinical practice (3rd ed.). Philadelphia, PA: Lippincott Williams \& Wilkins.

Thedon, T., Mandrick, K., Foissac, M., Mottet, D., \& Perrey, S. (2011). Degraded postural performance after muscle fatigue can be compensated by skin stimulation. Gait \& Posture, 33, 686-689. PubMed ID: 21454076 doi:10.1016/j.gaitpost.2011.02.027

Todorov, E. (2004). Optimality principles in sensorimotor control. Nature Neuroscience, 7 , 907-915. PubMed ID: 15332089 doi:10.1038/nn1309

Todorov, E., \& Jordan, M.I. (2002). Optimal feedback control as a theory of motor coordination. Nature Neuroscience, 5, 1226-1235. PubMed ID: 12404008 doi:10. $1038 / \mathrm{nn} 963$

Tseng, S., \& Morton, S. (2010). Impaired interlimb coordination of voluntary leg movements in poststroke hemiparesis. Journal of Neurophysiology, 104, 248-257. PubMed ID: 20463199 doi:10.1152/jn.00906.2009

Tsuji, T., Liu, M., Sonoda, S., Domen, K., \& Chino, N. (2000). The stroke impairment assessment set: Its internal consistency and predictive validity. Archives of Physical Medicine and Rehabilitation, 81, 863-868. PubMed ID: 10895996 doi:10.1053/apmr. 2000.6275

Vistamehr, A., Kautz, S.A., Bowden, M.G., \& Neptune, R.R. (2016). Correlations between measures of dynamic balance in individuals with post-stroke hemiparesis. Journal of Biomechanics, 49, 396-400. PubMed ID: 26795124 doi:10.1016/j.jbiomech.2015. 12.047

Walker, E.R., Hyngstrom, A.S., \& Schmit, B.D. (2016). Influence of visual feedback on dynamic balance control in chronic stroke survivors. Journal of Biomechanics, 49, 698-703. PubMed ID: 26916509 doi:10.1016/j.jbiomech.2016.01.028

Westlake, K.P., Wu, Y., \& Culham, E.G. (2007). Sensory-specific balance training in older adults: Effect on position, movement, and velocity sense at the ankle. Physical Therapy, 87, 560-568. PubMed ID: 17405803 doi:10.2522/ptj.20060262

Winter, D.A. (1990). Biomechanics and motor control of human movement. New York, NY: John Wiley \& Sons.

Wurdeman, S.R., Huben, N.B., \& Stergiou, N. (2012). Variability of gait is dependent on direction of progression: Implications for active control. Journal of Biomechanics, 45 , 653-659. PubMed ID: 22245103 doi:10.1016/j.jbiomech.2011.12.014 